\title{
BMJ Open Efficacy and safety of levetiracetam in children with epilepsy: protocol for an umbrella review of systematic reviews and meta-analyses of randomised controlled trials
}

Jing Gan, ${ }^{1,2}$ Dan Ma, ${ }^{1}$ Tao Xiong ${ }^{\oplus, 2,3}$

To cite: Gan J, Ma D, Xiong T. Efficacy and safety of levetiracetam in children with epilepsy: protocol for an umbrella review of systematic reviews and meta-analyses of randomised controlled trials. BMJ Open 2019;9:e029811. doi:10.1136/ bmjopen-2019-029811

- Prepublication history and additional material for this paper are available online. To view these files, please visit the journal online (http://dx.doi org/10.1136/bmjopen-2019029811).

Received 14 February 2019 Revised 17 May 2019 Accepted 14 June 2019

\section{Check for updates}

(C) Author(s) (or their employer(s)) 2019. Re-use permitted under CC BY-NC. No commercial re-use. See rights and permissions. Published by BMJ.

'Department of Pediatrics, West China Second University Hospital, Sichuan University, Chengdu, China

${ }^{2}$ Key Laboratory of Birth Defects and Related Diseases of Women and Children, Sichuan University, Ministry of Education, Chengdu,

China

${ }^{3}$ Deep Underground Space Medical Center, West China Hospital, Sichuan University, Chengdu, China

Correspondence to

Dr Tao Xiong;

tao_xiong@126.com

\section{ABSTRACT}

Introduction Epilepsy causes serious suffering in children and is associated with high morbidity and increased mortality. It impairs children's quality of life and places a heavy burden on healthcare resources. Levetiracetam has been used to prevent and treat paediatric epilepsy for years. To date, a number of systematic reviews have been performed to assess the efficacy and safety of levetiracetam in a variety of clinical settings. Conflicting outcomes have been reported for the same clinical issues. Our objective is to provide a comprehensive overview of the literature for clinicians and policymakers via an umbrella review that assesses the efficacy and safety of levetiracetam in children with epilepsy.

Methods and analysis We will follow the Joanna Briggs Institute's guidelines for umbrella reviews and the Preferred Reporting Items for Systematic Reviews and Meta-Analyses statement. The following seven databases will be searched from 1990 to February 2019: PubMed, Embase, Cochrane Database of Systematic Reviews, JBISRIR, EPPI, Epistemonikos and PROSPERO. We will provide evidence from existing systematic reviews and meta-analyses of randomised controlled trials regarding the use of levetiracetam in children with epilepsy. The intervention of interest is levetiracetam monotherapy and add-on therapies for prevention or treatment purposes. Studies will be individually selected and assessed by two reviewers. The primary outcomes of interest are epilepsy control, the efficacy of prophylaxis for provoked seizures and the mortality rate of children with epilepsy who received levetiracetam treatment. The secondary outcomes are adverse events and withdrawal rates due to adverse effects. The methodological quality of all reviews will be individually assessed by two reviewers using the 'A Measurement Tool to Assess Systematic Reviews' instrument. The Grading of Recommendations Assessment, Development and Evaluation assessment will be applied to evaluate the quality of evidence for each outcome of interest. A narrative description of an analysis of the systematic reviews will be tabulated to address objective and specific questions. Information from each review will be detailed in a table including the population, number of studies, total number of participants, year range of the trials, study designs of the primary trials, countries and settings of the trials, heterogeneity of results and assessment tools. Recommendations regarding each

\section{Strengths and limitations of this study}

- This protocol was designed following Joanna Briggs Institute's guidelines for umbrella reviews and reported following the Preferred Reporting Items for Systematic Reviews and Meta-Analyses statement.

- The methodological quality of all reviews will be individually assessed by two reviewers using the A Measurement Tool to Assess Systematic Reviews instrument, which evaluates the methods used in a review against 11 distinct criteria and assesses the degree to which the review methods are unbiased.

- The Grading of Recommendations Assessment, Development and Evaluation assessment will be used to evaluate the quality of evidence for each outcome of interest with the following criteria: quality of primary studies, design of primary studies, consistency and directness.

- The results of this review will inform clinicians and policy-makers of the efficacy and safety of levetiracetam in children with epilepsy.

- Only systemic reviews and meta-analyses of randomised controlled trials will be included.

outcome of levetiracetam will be categorised based on a protocol.

Ethics and dissemination This umbrella review will inform clinical and policy decisions regarding the efficacy and safety of levetiracetam for preventing and treating paediatric epilepsy. The results will be disseminated through a peerreviewed publication and conference presentations. Ethical approval is not required for this study.

\section{BACKGROUND}

Epilepsy is one of the most common neurological diseases globally. It affects more than 50 million people worldwide with a prevalence of $0.5 \%-1 \% .{ }^{1}$ People of all ages could be affected by epilepsy and there is a prominent distribution among children, especially in the age range from 1 to 12 years. ${ }^{2}$ Fortunately, epilepsyis one of the few treatable neurological 
diseases. Over $80 \%$ of children with new-onset epilepsy respond to the right treatment, and nearly $50 \%$ remain seizure-free after therapy is weaned. ${ }^{3}$ The remaining $20 \%$ of children are usually considered to have drug-resistant epilepsy and experience a greater risk for sudden unexpected death in epilepsy, adverse effects of anti-epileptic drugs and comorbidities such as neuropsychiatric problems, mood disorders, behavioural issues, attention deficits, psychosis and cognitive impairments. Drug-resistant epilepsy results in serious psychosocial, educational and vocational, as well as social, consequences, which often significantly reduces the quality of life of patients. ${ }^{45}$ Additionally, epileptic encephalopathies, embodying a variety of epilepsy syndromes such as Ohtahara syndrome, infantile spasms and Lennox-Gastaut syndrome, are able to cause profound nervous system sequelae such as developmental delay, cognitive impairments and intellectual diseases due to the epileptic activity. ${ }^{6}$ Moreover, neonatal seizures are special types of seizures with an incidence of $0.1 \%-0.3 \%$ for full-term babies and much higher in preterm babies. Neonatal seizures are associated with a very high risk of mortality and morbidity, including intellectual disability, mental retardation, cerebral palsy and autistic spectrum disorders. ${ }^{78}$ For infants, accumulating evidence demonstrates that prolonged febrile seizures are associated with hippocampal sclerosis and temporal lobe epilepsy. ${ }^{9}{ }^{10}$ Repetitive and prolonged provoked seizures resulting from trauma, tumour, parasites, high fever and craniotomy can increase the possibility of structural epilepsy and jointly contribute to the enormous global burden of epilepsy. ${ }^{11}$ Status epilepticus is the most extreme form of seizure with a mortality rate ranging between $3 \%$ and $40 \%$, which may be the highest among seizures or epilepsies. ${ }^{12}$ All these severe epilepsies in children will cause serious suffering for children, impair their quality of life and result in increased health costs. ${ }^{13}$

Anti-epileptic drugs are the cornerstone in the treatment of epilepsy. Levetiracetam is a second-generation anti-epileptic drug approved by the US Food and Drug Administration in 1999 for adjunctive therapy for focalonset epilepsy in adults. ${ }^{145}$ Although not clearly defined, the unique pharmacologic mechanism of levetiracetam works through binding to a protein called synaptic vesicle protein 2A, which reduces the rate of calcium-dependent vesicular neurotransmitter release. ${ }^{16}$ Levetiracetam is rapidly absorbed, highly bioavailable, not metabolised by the cytochrome P450 system and has a low protein binding rate, which means there is no competition for protein binding sites with other drugs. ${ }^{17}$ Two-thirds of the levetiracetam is excreted by the kidneys in its original form. Due to its efficacy and safety profile, levetiracetam has become popular worldwide and is available in three formulations (tablet, liquid solution and liquid injection). Currently, as add-on therapy, levetiracetam is approved for children 1 month and older with focal seizures, children 12 years and older with myoclonic seizures from juvenile myoclonic epilepsy and children 6 years and older with generalised tonic-clonic epilepsy. In
Europe, it is approved for the treatment of focal seizures as a monotherapy. Occasionally, levetiracetam has been used in an off-label manner to treat status epilepticus or as prophylaxis for seizures associated with neurological disorders. ${ }^{16} 18$ Additionally, levetiracetam has been widely used as prophylaxis for provoked seizures resulting from febrile, ${ }^{19} 20$ trauma,${ }^{21-23}$ aneurysm, ${ }^{24}$ tumour ${ }^{24} 25$ and craniotomy. ${ }^{24}{ }^{26}$ Regarding the dosage for infants and children, $40-60 \mathrm{mg} / \mathrm{kg}$ daily is recommended based on age. The most common adverse effects of levetiracetam include somnolence, dizziness, headache, coordination difficulty, irritability, infection, insomnia, nausea, loss of appetite, ataxia and loss of energy, which usually occur in the first month of therapy. ${ }^{27}$ Less common but serious side effects include severe skin reactions, suicidal thoughts or behaviour, death and birth defects. ${ }^{28}$ Behavioural problems are more frequently reported in children than in adults. ${ }^{29}$ However, levetiracetam is still regarded as one of the safest antiepileptic drugs.

\section{Why it is important to do this review}

Epilepsy ranked in the top four among the world's neurological disorders burden. ${ }^{30}$ The prevalence in children is quite high, and the treatment of epilepsy has practical significance in reducing the global health and socioeconomic burden. ${ }^{2}$ Levetiracetam has become one of the most important and commonly used drugs, as the 89th most prescribed medication in the USA in 2016 with more than 8 million prescriptions. There are an increasing number of prescriptions by paediatricians around the world. ${ }^{29} \mathrm{~A}$ number of systematic reviews regarding the efficacy and safety of levetiracetam in children with epilepsy have been published. When examining the same clinical issues, there have been contradictory outcomes. For example, the efficacy and safety of levetiracetam versus phenytoin for provoked seizure prophylaxis remains controversial. ${ }^{22-24} 31$ Whether levetiracetam plays the same role in different types of patients, such as newborns, children and adolescents, is also worthy of attention. Additionally, levetiracetam is widely used in an off-label manner as a monotherapy for epilepsy and seizures due to the narrow indication of the US Food and Drug Administration. This practice requires comprehensive evidence for further clinical guidance. To date, there is no 'overview' or umbrella review of systematic reviews or meta-analyses regarding the efficacy and safety of levetiracetam in children with epilepsy. There is an urgent need to systematically evaluate the benefit and potential harm of levetiracetam in children with epilepsy for better application of this drug. ${ }^{32}$ Therefore, we will use the most rigorous methods to summarise the currently known evidence from systematic reviews and meta-analyses of randomised controlled trials (RCTs).

\section{Is an overview the right approach?}

The following editorial decision tree from the Cochrane Comparing Multiple Interventions Methods Group will be applied. 
1. Only systematic reviews and meta-analyses will be selected for review, rather than individual trials.

2. Multiple interventions will not be compared with conclude the comparative effectiveness of these interventions.

3. The evidence from systematic reviews will be mapped without ranking the interventions.

Based on the above points, an overview is the right approach for this study as the editorial decision tree recommends.

\section{OBJECTIVES}

To systematically summarise the evidence from existing systematic reviews and meta-analyses of RCTs regarding the use of levetiracetam for preventing seizures and treating children with epilepsy. Specifically, the review will address the following questions:

1. The effectiveness of levetiracetam in children with epilepsy.

2. The safety of levetiracetam in children with epilepsy.

\section{Inclusion criteria}

\section{Participants}

The proposed umbrella review will consider systematic reviews and meta-analyses that include children with epilepsy aged $0-16$ years old. There will also be no restrictions on the types of epilepsy. All types of epilepsy will be searched, including but not limited to, generalised and focal epilepsies, motor and non-motor epilepsies, epileptic encephalopathy, refractory epilepsy, provoked seizures and status epilepticus.

\section{Interventions}

This review will consider systematic reviews and meta-analyses that relate to the use of levetiracetam in children with epilepsy, aiming to evaluate the efficacy and tolerability of levetiracetam. There will be no limitations based on dosing, formulation or mode of administration (intravenous, oral and so on).

We will include all levetiracetam therapies, including the following:

1. Levetiracetam used as a monotherapy and add-on therapy.

As a monotherapy, we will investigate the following comparisons.

- Levetiracetam only versus no treatment.

- Levetiracetam only versus sham treatment.

- Levetiracetam only versus another drug therapy.

- Levetiracetam only versus non-pharmacological therapy.

As an add-on therapy, we will investigate the following comparisons.

- Levetiracetam plus baseline treatment versus the same baseline treatment alone.

- Levetiracetam plus baseline treatment versus sham treatment plus the same baseline treatment.
- Levetiracetam plus baseline treatment versus other drugs plus the same baseline treatment.

- Levetiracetam plus baseline treatment versus non-pharmacological therapy plus the same baseline treatment.

2. Levetiracetam used both for the prevention of seizures and treatment of children with epilepsy.

\section{Comparator}

This umbrella review will consider systematic reviews and meta-analyses that compared the levetiracetam intervention to placebo, no treatment, other drugs or non-pharmacological therapy, such as ketogenic diet, vagus nerve stimulation therapy, deep brain stimulation therapy and surgery.

\section{Outcomes of interest}

This umbrella review will consider systematic reviews and meta-analyses that include the following outcome measures:

\section{Primary outcomes}

1. The efficacy of prophylaxis and treatment for epilepsy (both idiopathic and provoked seizures) outcomes, including (1) the absence of seizures, (2) a $50 \%$ responder rate after levetiracetam treatment and (3) seizure recurrence after the withdrawal of levetiracetam.

2. The efficacy of treatment for idiopathic epilepsy seizure outcomes, including (1) the absence of seizures, (2) a $50 \%$ responder rate after levetiracetam treatment and (3) seizure recurrence after the withdrawal of levetiracetam.

3. The efficacy of prophylaxis/treatment for provoked seizures resulting from fever, trauma, aneurysm, tumour, stroke and craniotomy.

\section{Secondary outcomes}

1. The total number and subgroups of adverse events: (1) most common and important adverse effects of levetiracetam including but not limited to, somnolence, dizziness, depression, hyperactivity, headache, coordination difficulty, irritability, severe skin reactions, suicidal thoughts or behaviour; (2) other adverse effects such as infection, loss of energy, insomnia, nausea, loss of appetite and ataxia and (3) mortality in children with epilepsy.

2. Withdrawal rate due to adverse effects.

3. Withdrawal rate due to lack of efficacy.

If the systematic review provided data regarding outcomes at different time points of follow-up, we will only use data for the longest follow-up.

\section{Context}

There will be no context constraints in this umbrella review.

\section{Types of studies}

This umbrella review will only enrol quantitative systematic reviews and meta-analyses with a clear and comprehensive 
search strategy and critical evaluation of bias risk. Only systematic reviews and meta-analyses with RCTs published in English will be considered. General literature reviews, critical reviews, narrative reviews, integrative reviews and primary research or solely qualitative reviews will be excluded from this umbrella review. Only the quantitative results, and not the qualitative results, will be extracted when incorporating mixed-method reviews.

\section{METHOD}

\section{Search strategy}

The search strategy is designed to retrieve both published and unpublished (grey literature) systemic reviews and meta-analyses of RCTs regarding the use of levetiracetam in children with epilepsy. There will be restrictions on the search strategy which will limit publications to those that were published in English and published between 1990 and February 2019. Endnote will be used to manage literature retrieval. A three-phase search strategy will be applied in this review. An initial limited search of PubMed was undertaken to identify the initial keywords, followed by analysis of the text words in the title and abstract and of the index terms used to describe each article, to develop a search strategy. A second search using the mature attached search strategy (online supplementary appendix I) will then be undertaken across all included databases: PubMed, Embase, Cochrane Database of Systematic Reviews, JBISRIR, EPPI, Epistemonikos and PROSPERO. Third, the reference lists of all identified reports and articles will be searched for additional studies. Databases such as Google Scholar will be searched for the grey literature.

Two independent experienced reviewers (JG and TX) will independently examine the titles and abstracts and select studies that need a review of the full article for eligibility assessment. Disagreements will be resolved through discussion or with the third reviewer (DM) by performing an additional independent evaluation.

Full-text studies failing to meet the inclusion criteria will be excluded and the reasons for exclusion will be described in detail. The search results will be reported in the final report with a Preferred Reporting Items for Systematic Reviews and Meta-analyses flow diagram. ${ }^{33}$

\section{Assessment of methodological quality}

Methodological quality of all reviews will be individually assessed by two reviewers using the instrument A Measurement Tool to Assess Systematic Reviews (AMSTAR), which evaluates the methods used in a review against 11 distinct criteria and assesses the degree to which review methods are unbiased. ${ }^{34}$ Each item on AMSTAR is rated as 'yes' (clearly done), 'no' (clearly not done), 'cannot answer' or 'not applicable' (online supplementary appendix II).

We will define the quality of each review based on the following cut-offs: scores of $0-3$ as low quality; scores of 4-7 as medium quality; scores of 8-10 as high quality; and adequately met all of the 11 criteria as the highest quality. A threshold score for inclusion is set as 4 , which means that reviews with low quality will be excluded. Consensus following disagreements will be achieved by a discussion between the two reviewers or with a third reviewer. If two or more studies were published within 2 years for the same intervention and same outcome, the study with the highest number of RCTs and the highest AMSTAR score will be included.

\section{Data extraction}

The JBI Data Extraction Form for Review for Systematic Reviews and Research Syntheses (online supplementary appendix III) will be used for data extraction from the eligible articles, ${ }^{35}$ within which the following characteristics of the studies will be described.

- Study details such as author/year/journal, objectives, participants (characteristics/total number), setting/context (cultural factors: ethnicity, socioeconomic status, minority group)and description of interventions.

- Search details such as the number of databases/ sources searched, date range of included studies, a detailed description of the included studies (number/type/country of origin of included studies).

- Appraisal instrument used and ratings.

- Analysis details such as the method of analysis, outcomes assessed, the results (including pooled effects (risk ratios, ORs or mean differences with 95\% CIs), significance, heterogeneity and comments.

Authors of the reviewed paper will be contacted in case of missing information to seek clarification.

\section{Subgroups}

If available, efficacy and safety estimates will be extracted separately for clinically important subgroups based on the types of participants (eg, newborns, infants, children and adolescents), types of epilepsies (eg, generalised epilepsy, focal epilepsy, refractory epilepsy, infantile spasms and provoked seizures) and modes of interventions (eg, monotherapy and add-on therapy, treatment and prevention strategy).

\section{Assessing certainty in the findings}

The Grading of Recommendations Assessment, Development and Evaluation (GRADE) assessment will be used to evaluate the quality of evidence for each outcome of interest with the following criteria: quality of primary studies, design of primary studies, consistency and directness. ${ }^{36}$ The GRADE approach categorises evidence from systematic reviews and meta-analyses into 'high', 'moderate', 'low' or 'very low' quality. Rather than being reassessed, the quality of evidence will be reported according to review authors' assessments by using the GRADE Pro 'Summary of findings' tables if provided, which will be collected during the data extraction process. Otherwise, the quality of evidence will be constructed by us using GRADE Pro Tool. 


\section{Data summary}

A narrative description of the analysis of the systematic reviews will be tabulated, describing the objective of the review and the specific questions addressed. The overall effect sizes will be presented. Information from each review will be detailed in a table including the population, number of studies, total number of participants, year range of those trials; study designs of the primary trials; countries and settings of those trials, heterogeneity of results and assessment tools. Pooled estimates will be reported if there is a high level of homogeneity. The recommendations of each outcome of levetiracetam will be categorised into one of the following five categories: (1) Effective or safe: indicating that the review found high-quality evidence of effectiveness or safety; (2) possibly effective or safe (more evidence needed): indicating that the review found moderate-quality evidence of effectiveness or safety, but more evidence is needed; (3) ineffective or harmful: indicating that the review found high-quality evidence of lack of effectiveness or high-quality evidence of adverse effects; (4) possibly ineffective or harmful: indicating that the review found moderate-quality evidence suggesting a lack of effectiveness or moderate-quality evidence of adverse effects for an intervention, but more evidence is needed and (5) no conclusions possible: indicating that the review found low- or very low-quality evidence or insufficient evidence to comment on the effectiveness or safety.

\section{Patient and public involvement}

This study is a systemic review and meta-analysis that involves no patients or the public.

\section{DISSEMINATION}

In this umbrella review, we will undertake a comprehensive review of previously published systematic reviews and meta-analyses assessing the efficacy and safety of levetiracetam for the prevention of seizures and treating children with epilepsy, by which we expect to draw a conclusion regarding the optimal levetiracetam treatment. The results of this study will be of interest to clinicians, policy-makers and patients. We plan to disseminate our findings through peer-reviewed journal publication and conference presentations. This review does not require ethical approval .

Acknowledgements The authors acknowledge suggestions from Mohan Pammi (Section of Neonatology, Department of Pediatrics, Baylor College of Medicine and Texas Children's Hospital, Houston, Texas, USA) on the conception and design of the analysis as well as suggested refinements to this manuscript.

Contributors The study was conceived by JG, DM and TX. JG and TX developed the eligibility criteria, search strategy, assessment of methodological quality, data extraction and data summary plan with guidance from DM. JG wrote the manuscript, to which all authors, DM, and TX, contributed.

Funding This work is supported by the National Science Foundation of China (No. 81501301 to Jing Gan, No. 81300525 to Tao Xiong) and Deep Underground Space Medical Center (No. DUGM201809 to Tao Xiong).

Competing interests None declared.
Patient consent for publication Not required.

Provenance and peer review Not commissioned; externally peer reviewed.

Open access This is an open access article distributed in accordance with the Creative Commons Attribution Non Commercial (CC BY-NC 4.0) license, which permits others to distribute, remix, adapt, build upon this work non-commercially, and license their derivative works on different terms, provided the original work is properly cited, appropriate credit is given, any changes made indicated, and the use is non-commercial. See: http://creativecommons.org/licenses/by-nc/4.0/.

\section{REFERENCES}

1. Lindy AS, Stosser MB, Butler E, et al. Diagnostic outcomes for genetic testing of 70 genes in 8565 patients with epilepsy and neurodevelopmental disorders. Epilepsia 2018;59:1062-71.

2. Minardi C, Minacapelli R, Valastro P, et al. Epilepsy in children: from diagnosis to treatment with focus on emergency. J Clin Med 2019;8:39.

3. Rosati A, De Masi S, Guerrini R. Antiepileptic drug treatment in children with epilepsy. CNS Drugs 2015;29:847-63.

4. Liu H, Yang Y, Wang Y, et al. Ketogenic diet for treatment of intractable epilepsy in adults: a meta-analysis of observational studies. Epilepsia Open 2018;3:9-17.

5. Tang F, Hartz AMS, Bauer B. Drug-resistant epilepsy: multiple hypotheses, few answers. Front Neurol 2017;8:301.

6. Howell KB, Harvey AS, Archer JS. Epileptic encephalopathy: Use and misuse of a clinically and conceptually important concept. Epilepsia 2016;57:343-7.

7. Pisani F, Facini C, Pavlidis E, et al. Epilepsy after neonatal seizures: literature review. Eur J Paediatr Neurol 2015;19:6-14.

8. Vasudevan C, Levene M. Epidemiology and aetiology of neonatal seizures. Semin Fetal Neonatal Med 2013;18:185-91.

9. Kasperaviciute D, Catarino CB, Matarin M, et al. Epilepsy, hippocampal sclerosis and febrile seizures linked by common genetic variation around SCN1A. Brain 2013;136(Pt 10):3140-50.

10. Lewis DV, Shinnar S, Hesdorffer DC, et al. Hippocampal sclerosis after febrile status epilepticus: the FEBSTAT study. Ann Neurol 2014;75:178-85.

11. Misra UK, Kalita J. Management of provoked seizure. Ann Indian Acad Neurol 2011;14:2-8.

12. Knake S, Hamer HM, Rosenow F. Status epilepticus: a critical review. Epilepsy \& behavior: E\&B 2009;15:10-14.

13. Allers K, Essue BM, Hackett ML, et al. The economic impact of epilepsy: a systematic review. BMC neurology 2015;15:245.

14. McCabe PH. New anti-epileptic drugs for the 21st century. Expert opinion on pharmacotherapy 2000;1:633-74.

15. Tidwell A, Swims M. Review of the newer antiepileptic drugs. Am J Manag Care 2003;9:253-76.

16. Kumar A, Kadian R. Levetiracetam. StatPearls: Treasure Island (FL): StatPearls Publishing 414 StatPearls Publishing LLC, 2018.

17. Ben-Menachem E. Levetiracetam: treatment in epilepsy. Expert Opin Pharmacother 2003;4:2079-88.

18. Crepeau AZ, Treiman DM. Levetiracetam: a comprehensive review. Expert Rev Neurother 2010;10:159-71.

19. Ly $\mathrm{H}$, Shi $\mathrm{XY}$, Li H, et al. Intermittent oral levetiracetam reduced recurrence of febrile seizure accompanied with epileptiform discharge: a pilot study. Italian journal of pediatrics 2018;44:70.

20. Ly H, Zou LP, Zhong JM, et al. Febrile seizure recurrence reduced by intermittent oral levetiracetam. Annals of clinical and translational neurology 2014;1:171-9.

21. Wilson CD, Burks JD, Rodgers RB, et al. Early and late posttraumatic epilepsy in the setting of traumatic brain injury: a meta-analysis and review of antiepileptic management. World neurosurgery 2018;110:e901-e6.

22. Yang $\mathrm{Y}$, Zheng $\mathrm{F}, \mathrm{Xu} \mathrm{X}$, et al. Levetiracetam versus phenytoin for seizure prophylaxis following traumatic brain injury: a systematic review and meta-analysis. CNS drugs 2016;30:677-88.

23. Jc X, Shen J, Shao WZ, et al. The safety and efficacy of levetiracetam versus phenytoin for seizure prophylaxis after traumatic brain injury: A systematic review and meta-analysis. Brain injury 2016;30:1054-61.

24. Lee $\mathrm{CH}, \mathrm{Koo} \mathrm{HW}$, Han SR, et al. Phenytoin versus levetiracetam as prophylaxis for postcraniotomy seizure in patients with no history of seizures: systematic review and meta-analysis. Journal of neurosurgery 2018:1-8.

25. Yuan Y, Peizhi Z, Maling G, et al. The efficacy of levetiracetam for patients with supratentorial brain tumors. J Clin Neurosci 2015;22:1227-31. 
26. Greenhalgh J, Weston J, Dundar Y, et al. Antiepileptic drugs as prophylaxis for postcraniotomy seizures. Cochrane Database Syst Rev 2018;5:CD007286.

27. Briggs DE, French JA. Levetiracetam safety profiles and tolerability in epilepsy patients. Expert opinion on drug safety 2004;3:415-24.

28. Chen B, Choi H, Hirsch LJ, et al. Psychiatric and behavioral side effects of antiepileptic drugs in adults with epilepsy. Epilepsy \& behavior: E\&B 2017;76:24-31.

29. Egunsola O, Choonara I, Sammons HM. Safety of levetiracetam in paediatrics: a systematic review. PloS one 2016;11:e0149686.

30. Beghi E. Addressing the burden of epilepsy: Many unmet needs. Pharmacological research 2016;107:79-84.

31. Bakr A, Belli A. A systematic review of levetiracetam versus phenytoin in the prevention of late post-traumatic seizures and survey of UK neurosurgical prescribing practice of antiepileptic medication in acute traumatic brain injury. British journal of neurosurgery 2018;32:237-44.
32. Aromataris E, Fernandez RS, Godfrey C, et al. Methodology for JBI umbrella reviews, 2014.

33. Moher D, Liberati A, Tetzlaff J, et al. Preferred reporting items for systematic reviews and meta-analyses: the PRISMA statement. Ann Intern Med 2009;151:264-9.

34. Shea BJ, Hamel C, Wells GA, et al. AMSTAR is a reliable and valid measurement tool to assess the methodological quality of systematic reviews. J Clin Epidemiol 2009;62:1013-20.

35. Aromataris E, Fernandez R, Godfrey CM, et al. Summarizing systematic reviews: methodological development, conduct and reporting of an umbrella review approach. Int J Evid Based Healthc 2015;13:132-40.

36. Guyatt GH, Oxman AD, Vist GE, et al. GRADE: an emerging consensus on rating quality of evidence and strength of recommendations. BMJ 2008;336:924-6. 